\title{
Several New Line Search Methods and Their Convergence
}

\author{
Zhenjun Shi ${ }^{1}$, Kimberly Kendricks ${ }^{1}$, Zhiwei $\mathrm{Xu}^{2}$, Yongning Tang ${ }^{3}$ \\ ${ }^{1}$ Department of Mathematics and Computer Science, Central State University, Wilberforce, USA \\ ${ }^{2}$ Department of Computer and Information Science, The University of Michigan, Dearborn, USA \\ ${ }^{3}$ School of Information Technology, Illinois State University, Normal, USA \\ Email: zshi@centralstate.edu, kkendricks@centralstate.edu, zwxu@umich.edu,ytang@ilstu.edu
}

Received September 28, 2012; revised January 7, 2013; accepted January 14, 2013

Copyright (C) 2013 Zhenjun Shi et al. This is an open access article distributed under the Creative Commons Attribution License, which permits unrestricted use, distribution, and reproduction in any medium, provided the original work is properly cited.

\begin{abstract}
In this paper, we propose several new line search rules for solving unconstrained minimization problems. These new line search rules can extend the accepted scope of step sizes to a wider extent than the corresponding original ones and give an adequate initial step size at each iteration. It is proved that the resulting line search algorithms have global convergence under some mild conditions. It is also proved that the search direction plays an important role in line search methods and that the step size approaches mainly guarantee global convergence in general cases. The convergence rate of these methods is also investigated. Some numerical results show that these new line search algorithms are effective in practical computation.
\end{abstract}

Keywords: Unconstrained Minimization; Line Search Method; Global Convergence; Convergence Rate

\section{Introduction}

Consider an unconstrained minimization problem

$$
\min f(x), x \in R^{n},
$$

where $R^{n}$ is an n-dimensional Euclidean space, $f: R^{n} \rightarrow R^{1}$ a continuously differentiable function. Throughout this paper, we use $g(x)=\nabla f(x)$ as the gradient function of $f(x)$. Given an initial point $x_{0}$, line search methods for solving (1) take the form

$$
x_{k+1}=x_{k}+\alpha_{k} d_{k}, k=0,1,2, \cdots,
$$

where $x_{k}$ is the current iterate, $d_{k}$ a search direction, and $\alpha_{k}$ a step size. Let $x^{*}$ be a minimizer of (1) and thus be a stationary point that satisfies $g\left(x^{*}\right)=0$. We denote $f\left(x_{k}\right)$ by $f_{k}, f\left(x^{*}\right)$ by $f^{*}$, and $\nabla f\left(x_{k}\right)$ by $g_{k}$, respectively.

In line search methods, there are two things to do at each iteration. One thing is to find a search direction $d_{k}$, and the other is to choose the step size $\alpha_{k}$ along the search direction $d_{k}$.

On the one hand, the search direction $d_{k}$ is generally required to satisfy the descent condition

$$
g_{k}^{T} d_{k}<0,
$$

which guarantees that $d_{k}$ is a descent direction of $f(x)$ at $x_{k}$. In order to ensure the global convergence of line search methods, we often require that the following condition holds,

$$
-\frac{g_{k}^{T} d_{k}}{\left\|g_{k}\right\| \cdot\left\|d_{k}\right\|} \geq C
$$

where $c \in(0,1]$ is a constant. The condition (4) is sometimes called the angle property (e.g., [1,2]). The choice of search direction $d_{k}$ plays an important role in designing line search methods (e.g., [3]). There are many techniques for choosing the search direction $d_{k}$ at the $k$ th iteration (e.g., [2,4,5]).

On the other hand, we should choose $\alpha_{k}$ to satisfy some line search rules. Line search rules can be classified into two types, exact line search rules and inexact line search rules. This paper is devoted to the case of inexact line search rules. There are three well-known inexact line search rules [6-9].

Armijo rule. Set scalars $s_{k}>0, \beta \in(0,1)$, and $\sigma \in(0,1 / 2)$. Choose $\alpha_{k}$ to be the largest $\alpha$ in $\left\{s_{k}, s_{k} \beta, s_{k} \beta^{2}, \cdots,\right\}$ such that

$$
f_{k}-f\left(x_{k}+\alpha d_{k}\right) \geq-\sigma \alpha g_{k}^{T} d_{k} .
$$

Remark. The original Armijo line search rule is to set $s_{k}=s$ with $s$ being a constant [8].

Goldstein rule. A fixed scalar $\sigma \in\left(0, \frac{1}{2}\right)$ is selected, 
and $\alpha_{k}$ is chosen to satisfy

$$
\sigma \leq \frac{f\left(x_{k}+\alpha_{k} d_{k}\right)-f_{k}}{\alpha_{k} g_{k}^{T} d_{k}} \leq 1-\sigma .
$$

It is possible to show that if $f$ is bounded below, then there exists an interval of step sizes $\alpha_{k}$ for which the relationships above are satisfied, and there are fairly simple algorithms for finding such a step size through a finite number of arithmetic operations.

Wolfe Rule. Choose $\alpha_{k}$ to satisfy

$$
f_{k}-f\left(x_{k}+\alpha_{k} d_{k}\right) \geq-\sigma \alpha_{k} g_{k}^{T} d_{k}
$$

and

$$
g\left(x_{k}+\alpha_{k} d_{k}\right)^{T} d_{k} \geq \gamma g_{k}^{T} d_{k}
$$

where $\sigma$ and $\gamma$ are some scalars with $\sigma \in\left(0, \frac{1}{2}\right)$ and $\gamma \in(\sigma, 1)$.

Lemma 1.1 ([1]) For the Wolfe rule, we assume that there is a scalar $M$ such that $f(x) \geq M$ for all $x \in R^{n}$. Let $\sigma \in\left(0, \frac{1}{2}\right)$ and $\gamma \in(\sigma, 1)$, and assume that $g_{k}^{T} d_{k}<0$. Then there exists an interval $\left[b_{1}, b_{2}\right]$ with $0<b_{1}<b_{2}$, such that every $\alpha \in\left[b_{1}, b_{2}\right]$ satisfies (7) and (8).

The above three line search rules can guarantee the existence of $\alpha_{k}$ under some mild conditions. However, how to find $\alpha_{k}$ is still a question. Especially, how to choose the initial step size $s_{k}$ in the Armijo rule is also very important in practical computation. In fact, how to solve the inequalities (6), (7), (8) is also a problem in computation. Some implementable modified Armijo rules were proposed [10-13]. Moreover, some nonmonotonic line search methods were also investigated [14-17]. However, can we find an approach to solve (6), (7), and (8) easily and economically? Sometimes, we first set an initial step size $s_{k}$ and substitute the test step size $\alpha=s_{k}$ into the inequalities (6), (7), or (8); if this $\alpha$ satisfies the inequalities, then we stop and find a step size $\alpha_{k}=\alpha$; otherwise, we need to use back-tracking or forward-tracking to adjust the test step size until we find an accepted step size $\alpha_{k}$.

In order to find $\alpha_{k}$ easily and economically, we need to solve three problems. One problem is how to estimate the initial step size $s_{k}$. The second problem is how to adjust the test step size when the test step size doesn't satisfy the inequalities. The third problem is whether we can extend the accepted scope of step sizes to a wider extent. Our research is focused on the second and third questions.

In this paper, we propose several line search rules for solving unconstrained minimization problems. The modi- fied line search rules used in the methods can extend the range of acceptable step sizes and give a suitable initial step size at each iteration. It is proved that the resulting line search methods have global convergence under some mild conditions. It is also proved that the search direction plays an important role in line search methods and that the step size rule mainly guarantees the global convergence in general cases. Numerical results show that the resulting algorithms are effective in practical computation.

The remainder of the paper is organized as follows. In Section 2, we describe the modified line search rules and its properties. In Section 3, we analyze the global convergence of resulting line search methods, and in Section 4 , we study further the convergence rate of the new line search methods. Numerical results are reported in Section 5.

\section{Modified Line Search Rules}

We first assume that

(H1). The function $f$ has a lower bound on $L_{0}=\left\{x \in R^{n} \mid f(x) \leq f\left(x_{0}\right)\right\}$, where $x_{0} \in R^{n}$ is given.

(H2). The gradient $g(x)=\nabla f(x)$ is uniformly continuous in an open convex set $B$ that contains $L_{0}$.

Sometimes, we further assume that

(H3). The gradient $g$ is Lipschitz continuous in an open convex set $B$ that contains $L_{0}$, i.e., there exists $L>0$ such that

$$
\|g(x)-g(y)\| \leq L\|x-y\|, \forall x, y \in B .
$$

It is apparent that (H3) implies (H2).

In the following three modified line search rules, we define

$$
w_{k}(\alpha)=\min \left\{\frac{1}{2} \alpha\left\|d_{k}\right\|,-\frac{g_{k}^{T} d_{k}}{\left\|d_{k}\right\|}\right\},
$$

where $\alpha \in(0,+\infty)$ is a variable.

Modified Armijo Rule. Set scalars $L_{k}>0, \beta \in(0,1)$ and $\sigma \in\left(0, \frac{1}{2}\right)$, and set $s_{k}=-\frac{g_{k}^{T} d_{k}}{L_{k}\left\|d_{k}\right\|^{2}}$. Let $\alpha_{k}$ be the largest $\alpha$ in $\left\{s_{k}, \beta s_{k}, \beta^{2} s_{k}, \cdots,\right\}$ such that

$$
f_{k}-f\left(x_{k}+\alpha d_{k}\right) \geq \sigma \alpha\left\|d_{k}\right\| w_{k}(\alpha) \text {. }
$$

Modified Goldstein Rule. A fixed scalar $\sigma \in\left(0, \frac{1}{2}\right)$ is selected, and $\alpha_{k}$ is chosen to satisfy

$$
-(1-\sigma) \alpha_{k} g_{k}^{T} d_{k} \geq f_{k}-f\left(x_{k}+\alpha_{k} d_{k}\right) \geq \sigma \alpha_{k}\left\|d_{k}\right\| w_{k}\left(\alpha_{k}\right) \text {. }
$$

Modified Wolfe Rule. The step size $\alpha_{k}$ is chosen to 
satisfy

$$
f_{k}-f\left(x_{k}+\alpha_{k} d_{k}\right) \geq \sigma \alpha_{k}\left\|d_{k}\right\| w_{k}\left(\alpha_{k}\right)
$$

and

$$
g\left(x_{k}+\alpha_{k} d_{k}\right)^{T} d_{k} \geq \gamma g_{k}^{T} d_{k}
$$

where $\sigma$ and $\gamma$ are some scalars with $\sigma \in\left(0, \frac{1}{2}\right)$ and $\gamma \in(\sigma, 1)$.

Apparently, if $\alpha_{k}$ satisfies (5), or (6), or (7) and (8), then $\alpha_{k}$ also satisfies (11), or (12), or (13) and (14). We may obtain the conclusion from the fact that

$$
\alpha_{k}\left\|d_{k}\right\| w_{k}\left(\alpha_{k}\right) \leq-\alpha_{k} g_{k}^{T} d_{k} .
$$

Therefore, if (H1) holds, then the three modified line searches are feasible. As a result, the modified line searches can extend the range of acceptable step sizes $\alpha_{k}$.

For the above three modified line search rules, we denote the three corresponding algorithms by Algorithm (NA), Algorithm (NG), and Algorithm (NW), respectively.

\section{Global Convergence}

In this section, we will prove that if (H1) and (H2) hold, $d_{k}$ satisfies (3), $\alpha_{k}$ is chosen so that (11), or (12), or (13) and (14) hold. The related algorithms generate an infinite sequence $\left\{x_{k}\right\}$, then

$$
\lim _{k \rightarrow+\infty} \frac{-g_{k}^{T} d_{k}}{\left\|d_{k}\right\|}=0 .
$$

Theorem 3.1 Assume that (H1), (H2), and (3) hold, $\left\{L_{k}\right\}$ satisfies $0<m_{0} \leq L_{k} \leq M_{0}$ with $m_{0}$ and $M_{0}$ being positive numbers. Algorithm (NA) with modified Armijo rule generates an infinite sequence $\left\{x_{k}\right\}$. Then (15) holds.

Proof. For Algorithm (NA), by setting

$$
K_{1}=\left\{k \mid \alpha_{k}=s_{k}\right\}, K_{2}=\left\{k \mid \alpha_{k}<s_{k}\right\},
$$

and by (11), we have

$$
\begin{aligned}
& f_{k}-f_{k+1} \geq s_{k} \sigma\left\|d_{k}\right\| w_{k}\left(s_{k}\right), \forall k \in K_{1}, \\
& f_{k}-f_{k+1} \geq \alpha_{k} \sigma\left\|d_{k}\right\| w_{k}\left(\alpha_{k}\right), \forall k \in K_{2} .
\end{aligned}
$$

Since $\alpha_{k}$ is the largest one to satisfy the modified Armijo rule, we will have $\alpha_{k}<\alpha_{k} / \beta \leq s_{k}, \forall k \in K_{2}$, and then $\alpha=\alpha_{k} / \beta$ makes the inequality sign of (11) change, i.e.,

$$
f_{k}-f\left(x_{k}+\alpha_{k} / \beta d_{k}\right)<\alpha_{k} \sigma\left\|d_{k}\right\| w_{k}\left(\alpha_{k} / \beta\right) / \beta, \forall k \in K_{2} .
$$

By the mean value theorem on the left-hand side of the above inequality, we can find $\theta_{k} \in[0,1]$ such that

$$
\begin{aligned}
& -\alpha_{k} g\left(x_{k}+\alpha_{k} \theta_{k} d_{k} / \beta\right)^{T} d_{k} / \beta \\
& =f_{k}-f\left(x_{k}+\alpha_{k} / \beta d_{k}\right) \\
& <\alpha_{k} \sigma\left\|d_{k}\right\| w_{k}\left(\alpha_{k} / \beta\right) / \beta \\
& \leq-\sigma \alpha_{k} g_{k}^{T} d_{k} / \beta, \quad \forall k \in K_{2} .
\end{aligned}
$$

Therefore,

$$
g\left(x_{k}+\theta_{k} \alpha_{k} d_{k} / \beta\right)^{T} d_{k}>\sigma g_{k}^{T} d_{k}, k \in K_{2} .
$$

By (H1), (3), and (11), it follows that $\left\{f_{k}\right\}$ is a nonincreasing number sequence and bounded from below, and it has a limit. Furthermore, we get from (11) that

$$
\sum_{k=0}^{\infty} \alpha_{k}\left\|d_{k}\right\| w_{k}\left(\alpha_{k}\right)<+\infty,
$$

and thus,

$$
\sum_{k \in K_{1}} s_{k}\left\|d_{k}\right\| w_{k}\left(s_{k}\right)+\sum_{k \in K_{2}} \alpha_{k}\left\|d_{k}\right\| w_{k}\left(\alpha_{k}\right)<+\infty .
$$

In order to prove (15), we use contrary proof to absurdity. Assume that there exists an $\epsilon>0$ and an infinite subset $K_{3} \subseteq\{0,1, \cdots\}$ such that

$$
-\frac{g_{k}^{T} d_{k}}{\left\|d_{k}\right\|} \geq \epsilon, \forall k \in K_{3} .
$$

Since $K_{1} \cup K_{2}=\{0,1, \cdots\}$, it is obvious that at least one of $K_{1} \cap K_{3}$ or $K_{2} \cap K_{3}$ is an infinite subset.

If $K_{1} \cap K_{2}$ is an infinite subset then by (21) and (20) we have

$$
\begin{aligned}
& \sum_{k \in K_{1} \cap K_{3}} \frac{\epsilon}{M_{0}} \min \left(\frac{\epsilon}{2 M_{0}}, \epsilon\right) \\
\leq & \sum_{k \in K_{1} \cap K_{3}} s_{k}\left\|d_{k}\right\| w_{k}\left(s_{k}\right) \\
\leq & \sum_{k \in K_{1}} s_{k}\left\|d_{k}\right\| w_{k}\left(s_{k}\right) \\
\leq & \sum_{k \in K_{1}} s_{k}\left\|d_{k}\right\| w_{k}\left(s_{k}\right)+\sum_{k \in K_{2}} \alpha_{k}\left\|d_{k}\right\| w_{k}\left(\alpha_{k}\right) \\
<+\infty . & <+\infty
\end{aligned}
$$

The contradiction shows that $K_{1} \cap K_{3}$ is not an infinite subset and $K_{2} \cap K_{3}$ must be an infinite subset.

By (21) and (20), we have

$$
\begin{aligned}
& \sum_{k \in K_{2} \cap K_{3}} \alpha_{k}\left\|d_{k}\right\| \min \left(\frac{1}{2} \alpha_{k}\left\|d_{k}\right\|, \epsilon\right) \\
& \leq \sum_{k \in K_{2} \cap K_{3}} \alpha_{k}\left\|d_{k}\right\| w_{k}\left(\alpha_{k}\right) \\
& \leq \sum_{k \in K_{2}} \alpha_{k}\left\|d_{k}\right\| w_{k}\left(\alpha_{k}\right) \\
& \leq \sum_{k \in K_{1}} s_{k}\left\|d_{k}\right\| w_{k}\left(s_{k}\right)+\sum_{k \in K_{2}} \alpha_{k}\left\|d_{k}\right\| w_{k}\left(\alpha_{k}\right) \\
&<+\infty .
\end{aligned}
$$

Therefore, 


$$
\alpha_{k}\left\|d_{k}\right\| \rightarrow 0\left(k \in K_{2} \cap K_{3}\right) .
$$

By the Cauchy-Schwartz inequality and (18), we obtain

$$
\begin{aligned}
& \left\|g\left(x_{k}+\alpha_{k} \theta_{k} d_{k} / \beta\right)-g_{k}\right\| \\
& =\frac{\left\|g\left(x_{k}+\alpha_{k} \theta_{k} d_{k} / \beta\right)-g_{k}\right\| \cdot\left\|d_{k}\right\|}{\left\|d_{k}\right\|} \\
& \geq \frac{\left(g\left(x_{k}+\alpha_{k} \theta_{k} d_{k} / \beta\right)-g_{k}\right)^{T} d_{k}}{\left\|d_{k}\right\|} \\
& \geq(1-\sigma) \frac{-g_{k}^{T} d_{k}}{\left\|d_{k}\right\|}, k \in K_{2} \cap K_{3} .
\end{aligned}
$$

By (H2), (22), and the above inequality, we have

$$
-\frac{g_{k}^{T} d_{k}}{\left\|d_{k}\right\|} \rightarrow 0\left(k \in K_{2} \cap K_{3}, k \rightarrow+\infty\right),
$$

which also contradicts (21). The contradiction shows that (15) holds.

Theorem 3.2 Assume that (H1), (H2), and (3) hold. Algorithm (NG) with the modified Goldstein line search generates an infinite sequence $\left\{x_{k}\right\}$. Then (15) holds.

Proof. By using the mean value theorem on the lefthand side inequality of (12), there exists $\theta_{k} \in[0,1]$ such that

$$
-\alpha_{k} g\left(x_{k}+\alpha_{k} \theta_{k} d_{k}\right)^{T} d_{k}=f_{k}-f_{k+1} \geq-(1-\sigma) \alpha_{k} g_{k}^{T} d_{k},
$$

and thus,

$$
g\left(x_{k}+\alpha_{k} \theta_{k} d_{k}\right)^{T} d_{k} \geq(1-\sigma) g_{k}^{T} d_{k} .
$$

By the right-hand side of (12) and (H1), it follows that $\left\{f_{k}\right\}$ is a monotone decreasing sequence and bounded below, and thus it has a limit. This shows that

$$
\sum_{k=0}^{\infty} \alpha_{k}\left\|d_{k}\right\| w_{k}\left(\alpha_{k}\right)<+\infty .
$$

Using the contrary proof, if (15) doesn't hold, then there exists an infinite subset $K \subseteq\{0,1, \cdots\}$ and an $\epsilon>0$ such that

$$
-\frac{g_{k}^{T} d_{k}}{\left\|d_{k}\right\|}>\epsilon, \forall k \in K .
$$

By (25) and (24), we obtain

$$
\begin{aligned}
& \sum_{k \in K} \alpha_{k}\left\|d_{k}\right\| \min \left(\frac{1}{2} \alpha_{k}\left\|d_{k}\right\|, \epsilon\right) \\
& \leq \sum_{k \in K} \alpha_{k}\left\|d_{k}\right\| w_{k}\left(\alpha_{k}\right) \\
& \leq \sum_{k=0}^{\infty} \alpha_{k}\left\|d_{k}\right\| w_{k}\left(\alpha_{k}\right)<+\infty,
\end{aligned}
$$

and thus,

$$
\alpha_{k}\left\|d_{k}\right\| \rightarrow 0(k \in K, k \rightarrow+\infty) .
$$

By the Cauchy-Schwartz inequality and (23), we have

$$
\begin{aligned}
& \left\|g\left(x_{k}+\alpha_{k} \theta_{k} d_{k}\right)-g_{k}\right\| \\
& =\frac{\left\|g\left(x_{k}+\alpha_{k} \theta_{k} d_{k}\right)-g_{k}\right\| \cdot\left\|d_{k}\right\|}{\left\|d_{k}\right\|} \\
& \geq \frac{\left(g\left(x_{k}+\alpha_{k} \theta_{k} d_{k}\right)-g_{k}\right)^{T} d_{k}}{\left\|d_{k}\right\|} \\
& \geq \sigma \frac{-g_{k}^{T} d_{k}}{\left\|d_{k}\right\|} .
\end{aligned}
$$

By (26) and (H2), and noting that

$\left\|\alpha_{k} \theta_{k} d_{k}\right\| \leq \alpha_{k}\left\|d_{k}\right\| \rightarrow 0(k \in K, k \rightarrow \infty)$ and the above inequality, we have

$$
\frac{-g_{k}^{T} d_{k}}{\left\|d_{k}\right\|} \rightarrow 0 \quad(k \in K, k \rightarrow+\infty),
$$

which contradicts (25). The contradiction shows that (15) holds.

Theorem 3.3 Assume that (H1), (H2), and (3) hold. Algorithm (NW) with modified Wolfe rule generates an infinite sequence $\left\{x_{k}\right\}$. Then (15) holds.

Proof. Using contrary proof, if (15) doesn't hold, then there exists an infinite subset $K \subseteq\{0,1, \cdots\}$ and an $\epsilon>0$ such that (25) holds. By (H1) and (13), it follows that (24) holds, and thus (26) holds.

By the Cauchy-Schwartz inequality and (14), we have

$$
\begin{aligned}
& \left\|g_{k+1}-g_{k}\right\| \\
& =\frac{\left\|g_{k+1}-g_{k}\right\| \cdot\left\|d_{k}\right\|}{\left\|d_{k}\right\|} \geq \frac{\left(g_{k+1}-g_{k}\right)^{T} d_{k}}{\left\|d_{k}\right\|} \geq(1-\gamma) \frac{-g_{k}^{T} d_{k}}{\left\|d_{k}\right\|} .
\end{aligned}
$$

By (H2), (26), and the above inequality, we have

$$
-\frac{g_{k}^{T} d_{k}}{\left\|d_{k}\right\|} \rightarrow 0(k \in K, k \rightarrow \infty),
$$

which is a contradiction to (25). The contradiction shows that (15) holds.

Theorem 3.4 Assume that (H1), (H3), and (3) hold, Algorithm (NA) with $0<m_{0} \leq L_{k} \leq M_{0}$, or Algorithm (NG), or Algorithm (NW) generates an infinite sequence $\left\{x_{k}\right\}$. Then there exists $k_{1} \in\{0,1, \cdots\}$ and $\eta_{0}>0$ such that

$$
f_{k}-f_{k+1} \geq \eta_{0}\left(\frac{-g_{k}^{T} d_{k}}{\left\|d_{k}\right\|}\right)^{2}, \forall k \geq k_{1} .
$$

Proof. Since (H3) implies (H2), the conclusions in Theorems 3.1, 3.2, and 3.3 are also true. We will use these conclusions and notations in the proofs of these theorems to our proof.

For Algorithm (NA), by (H3), the Cauchy-Schwartz 
inequality, and (18), we have

$$
\begin{aligned}
\alpha_{k} L\left\|d_{k}\right\|^{2} / \beta & \geq\left\|g\left(x_{k}+\alpha_{k} \theta d_{k} / \beta\right)-g_{k}\right\| \cdot\left\|d_{k}\right\| \\
& \geq\left(g\left(x_{k}+\alpha_{k} \theta d_{k} / \beta\right)-g_{k}\right)^{T} d_{k} \\
& >-(1-\sigma) g_{k}^{T} d_{k}, \quad k \in K_{2},
\end{aligned}
$$

where $K_{2}$ and $\theta_{k}$ are defined in the proof of Theorem 3.1. Thus,

$$
\alpha_{k}>-\beta(1-\sigma) \frac{g_{k}^{T} d_{k}}{L\left\|d_{k}\right\|^{2}}, k \in K_{2} .
$$

By (17), (28), and the proof of Theorem 3.1, we obtain

$$
f_{k}-f_{k+1} \geq \frac{\sigma \beta(1-\sigma)}{L} \min \left(\frac{\beta(1-\sigma)}{2 L}, 1\right) \cdot\left(\frac{-g_{k}^{T} d_{k}}{\left\|d_{k}\right\|}\right)^{2},
$$

where $k \in K_{2}, k \geq k_{1}$. By (16) and the proof of Theorem 3.1, we have

$$
f_{k}-f_{k+1} \geq \frac{\sigma}{M_{0}} \min \left(\frac{1}{2 M_{0}}, 1\right)\left(\frac{-g_{k}^{T} d_{k}}{\left\|d_{k}\right\|}\right)^{2}, k \in K_{1}, k \geq k_{1},
$$

where $K_{1}$ is defined in the proof of Theorem 3.1. Set

$$
\eta_{0}
$$

$$
=\sigma \min \left\{\frac{\beta-\beta \sigma}{L} \min \left(\frac{\beta-\beta \sigma}{2 L}, 1\right), \frac{1}{M_{0}} \min \left(\frac{1}{2 M_{0}}, 1\right)\right\},
$$

it follows that (27) holds.

For Algorithm (NG), by (H3), the Cauchy-Schwartz inequality, and (23), we have

$$
\begin{aligned}
\alpha_{k} L\left\|d_{k}\right\|^{2} & \geq\left\|g\left(x_{k}+\alpha_{k} \theta_{k} d_{k}\right)-g_{k}\right\| \cdot\left\|d_{k}\right\| \\
& \geq\left(g\left(x_{k}+\alpha_{k} \theta_{k} d_{k}\right)-g_{k}\right)^{T} d_{k} \\
& \geq-\sigma g_{k}^{T} d_{k},
\end{aligned}
$$

and thus,

$$
\alpha_{k} \geq-\frac{\sigma}{L} \frac{g_{k}^{T} d_{k}}{\left\|d_{k}\right\|^{2}} .
$$

By (12), (31), and Theorem 3.4, we have

$$
f_{k}-f_{k+1} \geq \frac{\sigma^{2}}{L} \min \left(\frac{\sigma}{2 L}, 1\right)\left(\frac{-g_{k}^{T} d_{k}}{\left\|d_{k}\right\|}\right)^{2}, k \geq k_{1} .
$$

By setting

$$
\eta_{0}=\frac{\sigma^{2}}{L} \min \left(\frac{\sigma}{2 L}, 1\right),
$$

it follows that (27) holds.

For Algorithm (NW), by (H3), the Cauchy-Schwartz inequality, and (14), we can prove that

$$
\alpha_{k} \geq-\frac{(1-\beta)}{L} \frac{g_{k}^{T} d_{k}}{\left\|d_{k}\right\|^{2}} .
$$

By (13), (32), and Theorem 3.1, we have

$f_{k}-f_{k+1} \geq \frac{\sigma(1-\beta)}{L} \min \left(\frac{1-\beta}{2 L}, 1\right)\left(\frac{-g_{k}^{T} d_{k}}{\left\|d_{k}\right\|}\right)^{2}, k \geq k_{1}$.

By setting

$$
\eta_{0}=\frac{\sigma(1-\beta)}{L} \min \left(\frac{1-\beta}{2 L}, 1\right),
$$

it follows that (27) holds.

Theorem 3.5 Assume that (H1), (H2), and (4) hold, Algorithm (NA) with $0<m_{0} \leq L_{k} \leq M_{0}$, or Algorithm (NG), or Algorithm (NW) generates an infinite sequence $\left\{x_{k}\right\}$. Then

$$
\lim _{k \rightarrow \infty}\left\|g_{k}\right\|=0 .
$$

Proof. By Theorems 3.1, 3.2, and 3.3, we obtain that (15) holds. By (4), when $k \rightarrow \infty$, we have

$$
c^{2}\left\|g_{k}\right\|^{2} \leq\left(\frac{-g_{k}^{T} d_{k}}{\left\|d_{k}\right\| \cdot\left\|g_{k}\right\|}\right)^{2} \cdot\left\|g_{k}\right\|^{2}=\left(\frac{-g_{k}^{T} d_{k}}{\left\|d_{k}\right\|}\right)^{2} \rightarrow 0 .
$$

Therefore, (15) holds.

Corollary 3.1 Assume that (H1), (H3), and (4) hold, Algorithm (NA) with $0<m_{0} \leq L_{k} \leq M_{0}$, or Algorithm (NG), or Algorithm (NW) generates an infinite sequence $\left\{x_{k}\right\}$. Then (34) holds.

Proof. By Theorem 3.5 and (4), we have

$$
\begin{aligned}
& f_{k}-f_{k+1} \geq \eta_{0}\left(\frac{-g_{k}^{T} d_{k}}{\left\|d_{k}\right\|}\right)^{2} \\
& =\eta_{0}\left(\frac{-g_{k}^{T} d_{k}}{\left\|d_{k}\right\| \cdot\left\|g_{k}\right\|}\right)^{2} \cdot\left\|g_{k}\right\|^{2} \geq \eta_{0} c^{2}\left\|g_{k}\right\|^{2} .
\end{aligned}
$$

By (H1) and the above inequality, we obtain that (34) holds.

Remark: Because (H3) implies (H2), it is obvious that the conditions of Corollary 3.1 imply the conditions of Theorem 3.5. Thus, Corollary 3.1 holds.

\section{Convergence Rate}

In order to analyze the convergence rate, we restrict our discussion to the case of uniformly convex objective functions. We further assume that

(H4): $f$ is twice continuously differentiable and uniformly convex on $R^{n}$.

Lemma 4.1 Assume that (H4) holds. Then (H1) and (H3) hold, $f(x)$ has a unique minimizer $x^{*}$, and there exists $0<m \leq M$ such that

$$
m\|y\|^{2} \leq y^{T} \nabla^{2} f(x) y \leq M\|y\|^{2}, \forall x, y \in R^{n} ;
$$




$$
\frac{1}{2} m\left\|x-x^{*}\right\|^{2} \leq f(x)-f\left(x^{*}\right) \leq \frac{1}{2} M\left\|x-x^{*}\right\|^{2}, \forall x \in R^{n} ;
$$

$$
M\|x-y\|^{2} \geq(g(x)-g(y))^{T}(x-y) \geq m\|x-y\|^{2},
$$

where $\forall x, y \in R^{n}$; and thus

$$
M\left\|x-x^{*}\right\|^{2} \geq g(x)^{T}\left(x-x^{*}\right) \geq m\left\|x-x^{*}\right\|^{2}, \forall x \in R^{n} \text {. }
$$

By (37) and (38), we can also obtain from the CauchySchwartz inequality that

$$
M\|x-y\| \geq\|g(x)-g(y)\| \geq m\|x-y\|
$$

and

$$
M\left\|x-x^{*}\right\| \geq\|g(x)\| \geq m\left\|x-x^{*}\right\|, \forall x \in R^{n} .
$$

Its proof can be seen from (e.g., [18,19], etc.). In this case, the Lipschitz constant $L$ of the gradient function $\nabla f(x)$ is $M$.

Theorem 4.1 Suppose that (H4) and (4) hold, any of the three algorithms generates an infinite sequence $\left\{x_{k}\right\}$. Then $\left\{x_{k}\right\}$ converges to $x^{*}$ at least $R$-linearly.

Proof. By Corollary 3.1 and Lemma 4.1, it follows that $\left\{x_{k}\right\}$ converges to $x^{*}$. By Theorem 3.5, (4) and (40), we have

$$
\begin{aligned}
f_{k}-f_{k+1} & \geq \eta_{0}\left(\frac{-g_{k}^{T} d_{k}}{\left\|d_{k}\right\|}\right)^{2} \\
& =\eta_{0}\left(-\frac{g_{k}^{T} d_{k}}{\left\|g_{k}\right\| \cdot\left\|d_{k}\right\|}\right)^{2}\left\|g_{k}\right\|^{2} \\
& \geq \eta_{0} c^{2}\left\|g_{k}\right\|^{2} \geq \eta_{0} c^{2} m^{2}\left\|x_{k}-x^{*}\right\|^{2} \\
& \geq 2 \frac{\eta_{0} c^{2} m^{2}}{M}\left(f_{k}-f\left(x^{*}\right)\right) .
\end{aligned}
$$

By the above inequality and setting $\rho=\sqrt{2 \frac{\eta_{0} c^{2} m^{2}}{M}}$, we can prove that $\rho<1$. In fact, by Theorem 3.5 and (39), and noting that $L=M$, we can obtain

$$
\eta_{0} \leq \frac{\sigma}{L}=\frac{\sigma}{M}
$$

and thus,

$$
\rho^{2}=\frac{2 \eta_{0} c^{2} m^{2}}{M} \leq \frac{2 \sigma c^{2} m^{2}}{M^{2}} \leq 2 \sigma c^{2}<1 .
$$

By setting $\theta=\sqrt{1-\rho^{2}}$, we have

$$
\begin{aligned}
& f_{k}-f\left(x^{*}\right) \leq\left(1-\rho^{2}\right)\left(f_{k-1}-f\left(x^{*}\right)\right) \\
& =\theta^{2}\left(f_{k-1}-f\left(x^{*}\right)\right) \leq \cdots \leq \theta^{2 k}\left(f_{0}-f\left(x^{*}\right)\right) .
\end{aligned}
$$

From the above first inequality, we can say that $\left\{f_{k}\right\}$ converges to $f^{*}$ at least $Q$-linearly. From the last inequality, we get that $\left\{f_{k}\right\}$ converges to $f^{*}$ at least $R$-linearly [18].

By setting $\omega=\sqrt{\frac{2\left(f_{0}-f\left(x^{*}\right)\right)}{m}}$, it follows that

$\left\|x_{k}-x^{*}\right\|^{2} \leq \frac{2}{m}\left(f_{k}-f\left(x^{*}\right)\right) \leq \frac{2\left(f_{0}-f\left(x^{*}\right)\right)}{m} \theta^{2 k}=\omega^{2} \theta^{2 k}$,

and thus

$$
\left\|x_{k}-x^{*}\right\| \leq \omega \theta^{k},
$$

which shows that $\left\{x_{k}\right\}$ converges to $x^{*}$ at least $R$-linearly [18].

\section{Numerical Results}

In the above sections, we investigated the theoretical properties and analyzed the global convergence and convergence rate of related line search methods under some mild conditions. In this section, we will study the numerical performance of algorithms with the new line search approaches.

First, we choose some numerical examples to test the Algorithms (NA), (NG), and (NW) and make some comparisons to the algorithms with the original line searches. The original line search methods are denoted by OA, OG, and OW, respectively.

The numerical examples are from [12]. We use the same symbols to denote the problems. For example, (P5) denotes Problem (P5) in [12]. For each problem, the limiting number of functional evaluations is set to 10,000 , and the stopping condition is

$$
\left\|g_{k}\right\| \leq 10^{-6} \text {. }
$$

We choose a portable computer with a Pentium 1.2 $\mathrm{MHz}$ CPU and Matlab 6.1 to test our algorithms. The parameters are set to $\sigma=0.38, \beta=0.87, \gamma=0.618$, $L_{0}=1$, and

$$
L_{k}=\frac{\left|\left(x_{k}-x_{k-1}\right)^{T}\left(g_{k}-g_{k-1}\right)\right|}{\left|x_{k}-x_{k-1}\right|^{2}},
$$

for $k \geq 1$.

Algorithm (NA): Step 0. Given parameters $\sigma=0.38$, $\beta=0.87$, and set $k:=0$;

Step 1. If $\left|g_{k}\right| \mid<10^{-6}$ then stop else taking $d_{k}=-g_{k}$

Step 2. Find step size $\alpha_{k}$ by using the modified Armijo rule;

Step 3. Set $x_{k+1}=x_{k}+\alpha_{k} d_{k}$ and set $k:=k+1$, go to Step 1.

For the Goldstein and the modified Goldstein rules, we use the following procedure to find the step size. 
Algorithm (NG): Step 0. Given parameters $\rho_{0}=2$ and $\rho=0.5$, set $k:=0$;

Step 1. If $\left|g_{k}\right| \mid<10^{-6}$ then stop else taking $d_{k}=-g_{k}$;

Step 2. Find step size $\alpha_{k}$ by using the following procedure:

Procedure 5.1. Set $\alpha=s_{k}=-\frac{g_{k}^{T} d_{k}}{L_{k}\left\|d_{k}\right\|^{2}}$ in which $L_{k}$ is defined by (43). Set two indices $d e c=0$ and inc $=0$.

Step 21. If $i n c * d e c=1$, then $\rho:=\sqrt{\rho}, \rho_{0}:=\sqrt{\rho_{0}}$, $\alpha_{k}=s_{k}$, and set inc $=0, \operatorname{dec}=0$;

Step 22. If (12) holds then $\alpha_{k}=\alpha$ and stop;

Step 23. If the right-hand inequality of (12) holds and the left doesn't hold, then $\alpha:=\rho_{0} \alpha$ and set inc $=1$, and go to Step 21;

Step 24. If the left-hand inequality of (12) holds and the right doesn't hold, then $\alpha:=\rho \alpha$ and set $\operatorname{dec}=1$, and go to Step 21;

Step 3. Set $x_{k+1}=x_{k}+\alpha_{k} d_{k}$ and set $k:=k+1$, go to Step 1.

For Wolfe and modified Wolfe rules, we use the following procedure to find the step size.

Algorithm (NW): Step 0. Given parameters $\rho_{0}=2$ and $\rho=0.5$, set $k:=0$;

Step 1. If $\left|g_{k}\right| \mid<10^{-6}$ then stop else taking $d_{k}=-g_{k}$;
Step 2. Find step size $\alpha_{k}$ by using the following procedure:

Procedure 5.2. Set $\alpha=s_{k}=-\frac{g_{k}^{T} d_{k}}{L_{k}\left\|d_{k}\right\|^{2}}$ in which $L_{k}$ is defined by (43). Set two indices $d e c=0$ and inc $=0$.

Step 21. If inc $* \operatorname{dec}=1$, then $\rho:=\sqrt{\rho}, \rho_{0}:=\sqrt{\rho_{0}}$, $\alpha_{k}=s_{k}$, and set inc $=0$, dec $=0$;

Step 22. If (13) and (14) hold, then $\alpha_{k}=\alpha$ and stop;

Step 23. If (13) holds and (14) doesn't hold, then $\alpha:=\rho_{0} \alpha$ and set inc $=1$, and go to Step 21;

Step 24. If (14) holds and (13) doesn't hold, then $\alpha:=\rho \alpha$ and set $\operatorname{dec}=1$, and go to Step 21;

Step 3. Set $x_{k+1}=x_{k}+\alpha_{k} d_{k}$ and set $k:=k+1$, go to Step 1.

In numerical experiments, we take $d_{k}=-g_{k}$.

We can see from Table 1 that the modified Wolfe line approach is the best one in practical computation. In fact, NA, NG and NW are all better than OA, OG, and OW. This shows that the modified line searches are effective in practical computation and significantly reduce the number of functional evaluations and iterations when reaching the same precision. Moreover, we found that the new modified line approaches can be used to any descent methods. For example, we can take quasi-Newton direction $d_{k}=-H_{k} g_{k}$ in the line search methods and use these modified line search approaches to find a step size.

Table 1. The number of iterations and functional evaluations.

\begin{tabular}{|c|c|c|c|c|c|c|c|}
\hline $\mathrm{P}$ & $\mathrm{n}$ & $\mathrm{OA}$ & OG & OW & NA & NG & NW \\
\hline P5 & 2 & $8 / 12$ & $7 / 11$ & $7 / 10$ & $6 / 7$ & $6 / 7$ & $6 / 7$ \\
\hline $\mathrm{P} 13$ & 4 & 23/35 & $21 / 32$ & $20 / 29$ & $17 / 20$ & $16 / 21$ & $15 / 22$ \\
\hline $\mathrm{P} 14$ & 4 & $36 / 52$ & $32 / 48$ & $32 / 48$ & 23/32 & $23 / 35$ & $21 / 29$ \\
\hline P16 & 4 & $14 / 63$ & $16 / 67$ & $14 / 62$ & $16 / 43$ & $17 / 51$ & $11 / 29$ \\
\hline P20 & 9 & $12 / 17$ & $12 / 19$ & $13 / 15$ & $11 / 14$ & $11 / 14$ & $11 / 12$ \\
\hline P21 & 16 & $18 / 25$ & $18 / 27$ & $18 / 26$ & $12 / 24$ & $12 / 27$ & $11 / 20$ \\
\hline $\mathrm{P} 23$ & 8 & $30 / 41$ & $33 / 49$ & $31 / 45$ & $25 / 34$ & $23 / 36$ & $24 / 32$ \\
\hline $\mathrm{P} 24$ & 20 & $52 / 64$ & $55 / 71$ & $50 / 58$ & $35 / 46$ & $33 / 42$ & 28/38 \\
\hline P25 & 50 & 11/123 & 12/118 & $10 / 67$ & $11 / 21$ & $12 / 23$ & $11 / 18$ \\
\hline P26 & 50 & $14 / 30$ & $16 / 42$ & $15 / 38$ & $12 / 28$ & $12 / 24$ & $11 / 19$ \\
\hline P30 & 20 & $13 / 22$ & $13 / 26$ & $13 / 23$ & $11 / 26$ & $11 / 21$ & $10 / 18$ \\
\hline $\mathrm{P} 21$ & 1000 & 98/563 & 89/483 & $76 / 428$ & $67 / 310$ & $54 / 258$ & $48 / 211$ \\
\hline P21 & 5000 & $143 / 721$ & $126 / 565$ & $118 / 475$ & $76 / 426$ & 78/384 & $68 / 236$ \\
\hline P23 & 1000 & $120 / 798$ & 117/695 & $120 / 572$ & $73 / 275$ & $76 / 248$ & $64 / 198$ \\
\hline P23 & 5000 & $185 / 775$ & $167 / 883$ & $151 / 663$ & $68 / 85$ & $61 / 83$ & $42 / 65$ \\
\hline CPU & - & $189 \mathrm{~s}$ & $198 \mathrm{~s}$ & $135 \mathrm{~s}$ & $121 \mathrm{~s}$ & $117 \mathrm{~s}$ & $93 \mathrm{~s}$ \\
\hline
\end{tabular}


If we take $L_{k}$ like $L_{0}=1$, and

$$
L_{k}=\frac{\left\|g_{k}-g_{k-1}\right\|}{\left\|x_{k}-x_{k-1}\right\|},
$$

for $k \geq 1$, and use (44) to replace (43) in Procedure 5.2, we have the results in Table 2. From Table 3, we found that the estimation (43) seems better that (44) in many situations. Actually, by Cauchy-Schwartz inequality, we

Table 2. The number of iterations and functional evaluations.

\begin{tabular}{|c|c|c|c|c|c|c|c|}
\hline $\mathrm{P}$ & $\mathrm{n}$ & $\mathrm{OA}$ & OG & OW & NA & NG & NW \\
\hline P5 & 2 & $8 / 12$ & $7 / 11$ & $7 / 10$ & 7/11 & $7 / 12$ & $7 / 11$ \\
\hline $\mathrm{P} 13$ & 4 & 23/35 & 21/32 & $20 / 29$ & $19 / 23$ & $18 / 25$ & $18 / 25$ \\
\hline P14 & 4 & $36 / 52$ & $32 / 48$ & $32 / 48$ & $25 / 37$ & $25 / 37$ & $27 / 31$ \\
\hline P16 & 4 & $14 / 63$ & $16 / 67$ & $14 / 62$ & $16 / 45$ & $18 / 53$ & $12 / 32$ \\
\hline P20 & 9 & $12 / 17$ & $12 / 19$ & $13 / 15$ & $11 / 18$ & $11 / 19$ & $12 / 19$ \\
\hline $\mathrm{P} 21$ & 16 & $18 / 25$ & $18 / 27$ & $18 / 26$ & $14 / 31$ & $12 / 25$ & $14 / 28$ \\
\hline P23 & 8 & $30 / 41$ & $33 / 49$ & $31 / 45$ & $26 / 37$ & $25 / 37$ & $26 / 38$ \\
\hline $\mathrm{P} 24$ & 20 & $52 / 64$ & $55 / 71$ & $50 / 58$ & $39 / 48$ & $32 / 45$ & $29 / 43$ \\
\hline P25 & 50 & 11/123 & $12 / 118$ & $10 / 67$ & $11 / 32$ & $12 / 34$ & $12 / 28$ \\
\hline P26 & 50 & $14 / 30$ & $16 / 42$ & $15 / 38$ & $12 / 35$ & $13 / 44$ & $14 / 27$ \\
\hline P30 & 20 & $13 / 22$ & $13 / 26$ & $13 / 23$ & $12 / 27$ & $13 / 26$ & $12 / 26$ \\
\hline $\mathrm{P} 21$ & 1000 & 98/563 & 89/483 & $76 / 428$ & $69 / 354$ & $58 / 263$ & $53 / 265$ \\
\hline $\mathrm{P} 21$ & 5000 & $143 / 721$ & $126 / 565$ & $118 / 475$ & 78/433 & $81 / 420$ & $73 / 263$ \\
\hline P23 & 1000 & $120 / 798$ & $117 / 695$ & $120 / 572$ & $94 / 321$ & $85 / 291$ & $69 / 238$ \\
\hline P23 & 5000 & $185 / 775$ & $167 / 883$ & $151 / 663$ & $72 / 120$ & $63 / 72$ & $43 / 69$ \\
\hline CPU & - & $189 s$ & $198 \mathrm{~s}$ & $135 \mathrm{~s}$ & $132 \mathrm{~s}$ & 141s & $128 \mathrm{~s}$ \\
\hline
\end{tabular}

Table 3. The number of iterations and functional evaluations.

\begin{tabular}{|c|c|c|c|c|c|c|c|}
\hline $\mathrm{P}$ & $\mathrm{n}$ & OA & OG & OW & NA & NG & NW \\
\hline ARWHEAD & $10^{4}$ & $46 / 198$ & $42 / 137$ & $32 / 120$ & $26 / 64$ & $25 / 47$ & $25 / 54$ \\
\hline DQDRTIC & $10^{4}$ & $18 / 43$ & 17/38 & $15 / 32$ & $16 / 31$ & $15 / 43$ & $14 / 28$ \\
\hline ENGVAL1 & $10^{4}$ & $18 / 36$ & $17 / 29$ & $15 / 28$ & $12 / 25$ & $12 / 25$ & $12 / 25$ \\
\hline VAREIGVL & $10^{4}$ & $21 / 28$ & $18 / 32$ & $17 / 31$ & $16 / 25$ & $17 / 32$ & $15 / 28$ \\
\hline WOODS & $10^{4}$ & $19 / 29$ & $17 / 26$ & $15 / 24$ & $17 / 32$ & $16 / 43$ & $16 / 41$ \\
\hline LIARWHD & $10^{4}$ & $32 / 76$ & $35 / 88$ & $31 / 45$ & $28 / 46$ & $28 / 52$ & $28 / 34$ \\
\hline MOREBV & $10^{4}$ & $76 / 86$ & $72 / 124$ & 73/97 & $72 / 97$ & $72 / 97$ & $60 / 81$ \\
\hline NONDIA & $10^{4}$ & $32 / 44$ & $28 / 52$ & $26 / 42$ & $22 / 36$ & $25 / 48$ & $23 / 34$ \\
\hline TQUARTIC & $10^{4}$ & $29 / 43$ & 27/38 & $25 / 31$ & $24 / 36$ & $24 / 43$ & $24 / 43$ \\
\hline POWELLSG & $10^{4}$ & $57 / 126$ & $59 / 119$ & $53 / 97$ & $48 / 126$ & $53 / 92$ & $42 / 77$ \\
\hline QUARTC & $10^{4}$ & $53 / 127$ & $42 / 116$ & $34 / 98$ & $32 / 74$ & $33 / 68$ & $31 / 66$ \\
\hline SCHMVETT & $10^{4}$ & $28 / 75$ & 28/92 & $25 / 82$ & $24 / 38$ & $25 / 42$ & $23 / 38$ \\
\hline SPARSQUR & $10^{4}$ & $54 / 93$ & $47 / 110$ & $48 / 90$ & $42 / 73$ & $45 / 73$ & $40 / 65$ \\
\hline SROSENBR & $10^{4}$ & $31 / 95$ & 29/85 & $25 / 82$ & 29/66 & $25 / 78$ & $24 / 64$ \\
\hline TOINTGSS & $10^{4}$ & $26 / 67$ & 28/73 & $26 / 75$ & $24 / 56$ & $21 / 52$ & $24 / 48$ \\
\hline CPU & & $125 \mathrm{~s}$ & $116 \mathrm{~s}$ & $108 \mathrm{~s}$ & $97 \mathrm{~s}$ & $88 \mathrm{~s}$ & $72 \mathrm{~s}$ \\
\hline
\end{tabular}


have

$$
\frac{\left|\left(x_{k}-x_{k-1}\right)^{T}\left(g_{k}-g_{k-1}\right)\right|}{\left\|x_{k}-x_{k-1}\right\|^{2}} \leq \frac{\left\|g_{k}-g_{k-1}\right\|}{\left\|x_{k}-x_{k-1}\right\|},
$$

which shows that (43) can produce a larger initial step size $s_{k}$ than (44) does.

In fact, in the beginning of iterations, larger step size can quicken the convergence of resulting algorithms for solving well conditioned middle scale problems. For large scale or even ill-conditioned problems, what is the performance of the resulting algorithms with new modified line search rules? We chose standard test problems from [20-22] to test the new modified line search rules. The numerical results are reported in Table 3.

For large scale problems, numerical results show that the resulting algorithms with the modified line search rules are more effective than the original ones in many cases. The reason is that step size estimation is more useful for such large scale problems that have sparse Hessian matrix. Larger step size plays an important role in the convergence of resulting algorithms with modified line searches. Therefore, initial step size estimation and extending the range of acceptable step sizes is necessary in line search design and algorithm design.

\section{Conclusions}

We proposed several new line search algorithms for solving unconstrained minimization problems. The modified line search rules used in the methods can extend the range of acceptable step sizes to a wider extent and give a suitable initial step size at each iteration. It is clarified that the resulting line search algorithms have global convergence under weak conditions. It is also proved that the search direction plays an important role in these methods and that the step size mainly guarantees global convergence in some cases. The convergence rate of these algorithms is investigated. These theoretical results can help us design new algorithms in practice. Furthermore, we extended the line search methods theoretically in some broad sense. Numerical results show that these new modified line search rules are useful and effective in practical computation.

For the future research, we should study the numerical performance of special line search methods for largescale practical problems. Moreover, we can generalize these modified line search approaches to nonmonotone cases $[14,16]$. We can also investigate step-size in different ways [23-25].

\section{Acknowledgements}

The authors are very grateful to the referees and editors for their helpful and valuable comments and suggestions that greatly improved the paper.

\section{REFERENCES}

[1] D. P. Bertsekas, "Constrained Optimization and Lagrange Multiplier Methods,” Academic Press Inc., Waltham, 1982.

[2] J. Nocedal and J. W. Stephen, "Numerical Optimization,” Springer-Verlag New York, Inc., New York, 1999. doi:10.1007/b98874

[3] Y. G. Evtushenko, "Numerical Optimization Techniques," Optimization Software Inc., Publications Division, New York, 1985.

[4] J. P. Dussault, "Convergence of Implementable Descent Algorithms for Unconstrained Optimization,” Journal of Optimization Theory and Applications, Vol. 104, No. 3, 2000, pp. 749-750. doi:10.1023/A:1004602012151

[5] B. Rhanizar, "Hybrid Procedures for Solving Some Unconstrained Nonlinear Optimization Problems, Applied Numerical Mathematics, Vol. 30, No. 4, 1999, pp. 459474. doi:10.1016/S0168-9274(98)00068-3

[6] L. Armijo, "Minimization of Functions Having Lipschitz Continuous First Partial Derivatives," Pacific Journal of Mathematics, Vol. 16, No. 1, 1966, pp. 1-3. doi:10.2140/pjm.1966.16.1

[7] A. A. Goldstein, “On Steepest Descent Method,” Journal on Society for the Industrial and Applied Mathematics Series A Control, Vol. 3, No. 1, 1965, pp. 147-151.

[8] W. Y. Sun and Y. X. Yuan, "Optimization Theory and Methods-Nonlinear Programming,” Springer Optimization and Its Applications, Vol. 1. Springer, New York, 2006.

[9] P. Wolfe, "Convergence Conditions for Ascent Methods," SIAM Review, Vol. 11, No. 2, 1969, pp. 226-235. doi:10.1137/1011036

[10] Z. J. Shi, "Convergence of Line Search Metods for Unconstrained Optimization," Applied Mathematics and Computation, Vol. 157, No. 2, 2004, pp. 393-405. doi:10.1016/j.amc.2003.08.058

[11] Z. J. Shi and J. Shen, "Convergence of Descent Method without Line Search,” Applied Mathematics and Computation, Vol. 167, No. 1, 2005, pp. 94-107. doi:10.1016/j.amc.2004.06.097

[12] Z. J. Shi and J. Shen, "New Inexact Line Search Method for Unconstrained Optimization," Journal of Optimization Theory and Applications, Vol. 127, No. 2, 2005, pp. 425-446. doi:10.1007/s10957-005-6553-6

[13] Z. J. Shi and J. Shen, "Step Size Estimation for Unconstrained Optimization Methods," Journal of Computational and Applied Mathematics, Vol. 24, No. 3, 2005, pp. 399-417.

[14] Y. H. Dai, “On the Nonmonotone Line Search,” Journal of Optimization Theory and Applications, Vol. 112, No. 2, 2002, pp. 315-330. doi:10.1023/A:1013653923062

[15] Z. J. Shi and J. Shen, "Convergence of Nonmonotone Line Search Method,” Journal of Computational and Applied Mathematics, Vol. 193, No. 2, 2006, pp. 397-412. doi:10.1016/j.cam.2005.06.033 
[16] W. Y. Sun, J. Y. Han and J. Sun, “Global Convergence of Nonmonotone Descent Methods for Unconstrained Optimization Problems," Journal of Computational and Applied Mathematics, Vol. 146, No. 1, 2002, pp. 89-98. doi:10.1016/S0377-0427(02)00420-X

[17] H. C. Zhang and W. W. Hager, "A Nonmonotone Line Search Technique and Its Application to Unconstrained Optimization," The SIAM Journal on Optimization, Vol. 14, No. 4, 2004, pp. 1043-1056. doi:10.1137/S1052623403428208

[18] J. M. Ortega and W. C. Rheinboldt, "Iterative Solution of Nonlinear Equations in Several Variables," Academic Press, Waltham, 1970.

[19] R. T. Rockafellar, “Convex Analysis,” Princeton University Press, Princeton, 1970.

[20] I. Bongartz, A. R. Conn, N. I. M. Gould and P. L. Toint, "CUTE: Constrained and Unconstrained Testing Environments," ACM Transactions on Mathematical Software, Vol. 21, No. 1, 1995, pp. 123-160. doi:10.1145/200979.201043

[21] W. W. Hager and H. C. Zhang, “A New Conjugate Gra- dient Method with Guaranteed Descent and an Efficient Line Search,” The SIAM Journal on Optimization, Vol. 16, No. 1, 2005, pp. 170-192. doi:10.1137/030601880

[22] W. W. Hager and H. C. Zhang, “Algorithm 851: CG DESCENT, A Conjugate Gradient Method with Guaranteed Descent," ACM Transactions on Mathematical Software, Vol. 32, No. 1, 2006, pp. 113-137. doi:10.1145/1132973.1132979

[23] A. I. Cohen, "Stepsize Analysis for Descent Methods," Journal of Optimization Theory and Applications, Vol. 33, No. 2, 1981, pp. 187-205. doi:10.1007/BF00935546

[24] J. C. Gilbert and J. Nocedal, "Global Convergence Properties of Conjugate Gradient Methods for Optimization,” The SIAM Journal on Optimization, Vol. 2, No. 1, 1992, pp. 21-42. doi:10.1137/0802003

[25] Z. J. Shi, “A New Memory Gradient Method under Exact Line Search,” Asia-Pacific Journal of Operational Research, Vol. 20, No. 2, 2003, pp. 275-284. 